\title{
Hydrogenation-driven phase transition in single-layer $\mathrm{TiSe}_{2}$
}

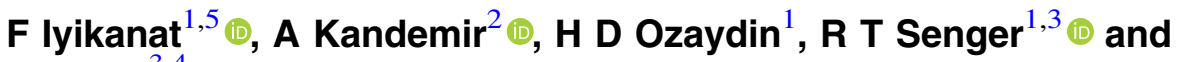 \\ H Sahin ${ }^{3,4}$ (1) \\ ${ }^{1}$ Department of Physics, Izmir Institute of Technology, 35430, Izmir, Turkey \\ ${ }^{2}$ Department of Materials Science and Engineering, Izmir Institute of Technology, 35430, Izmir, Turkey \\ ${ }^{3}$ ICTP-ECAR Eurasian Center for Advanced Research, Izmir Institute of Technology, 35430, Izmir, \\ Turkey \\ ${ }^{4}$ Department of Photonics, Izmir Institute of Technology, 35430, Izmir, Turkey \\ E-mail: fadiliyikanat@iyte.edu.tr and hasansahin@iyte.edu.tr
}

Received 12 September 2017

Accepted for publication 19 October 2017

Published 15 November 2017

\begin{abstract}
First-principles calculations based on density-functional theory are used to investigate the effects of hydrogenation on the structural, vibrational, thermal and electronic properties of the charge density wave (CDW) phase of single-layer $\mathrm{TiSe}_{2}$. It is found that hydrogenation of single-layer $\mathrm{TiSe}_{2}$ is possible through adsorption of a $\mathrm{H}$ atom on each Se site. Our total energy and phonon calculations reveal that a structural phase transition occurs from the CDW phase to the $T_{d}$ phase upon full hydrogenation. Fully hydrogenated $\mathrm{TiSe}_{2}$ presents a direct gap semiconducting behavior with a band gap of $119 \mathrm{meV}$. Full hydrogenation also leads to a significant decrease in the heat capacity of single-layer $\mathrm{TiSe}_{2}$.
\end{abstract}

Keywords: first-principles, monolayer, phase transition, heat capacity, hydrogenation

(Some figures may appear in colour only in the online journal)

\section{Introduction}

After the discovery of graphene, [1,2] two-dimensional (2D) materials have aroused considerable interest in recent years. The family of synthesized 2D crystals is rapidly expanding by the aid of improved experimental techniques. Over the past few years, vast number of $2 \mathrm{D}$ materials such as hexagonal boron nitride, [3-5] silicene, [6, 7] transition metal dichalcogenides (TMDs), [8-13] transition metal trichalcogenides (TMTs), [14-16] were synthesized in few-layer and even single-layer forms. Among these materials, TMDs became the focus of interest due to their wide variety of chemical compositions and their electronic and optical properties.

TMDs exhibit desirable electronic properties, such as high carrier mobility, [17, 18] low effective mass, [19] layer-dependent tunable band gap, [20] and direct-to-indirect band gap crossover [9, 21]. Different $d$-electron counts of metal atoms in TMDs, results in a range of electronic properties including metallic, insulating, semiconducting and even superconducting.

5 Author to whom any correspondence should be addressed.
TMDs have a lamellar crystal structure where the layers are held together by weak van der Waals (vdW) forces, while the metals and chalcogens are covalently bonded within the layer. The large family of TMDs presents promising materials for the design of field-effect transistors, light harvesting devices, ultrasensitive chemical sensors, and flexible electronic devices.

One of the recently synthesized single layer TMDs is $\mathrm{TiSe}_{2}$ [22]. $\mathrm{TiSe}_{2}$ has an octahedral crystal structure with vdW stacked layers. Although there are several experimental studies to determine whether the electronic structure of bulk $\mathrm{TiSe}_{2}$ is semiconducting or semimetallic, the exact electronic structure of it remains controversial. Among TMDs, bulk $\mathrm{TiSe}_{2}$ has sparked particular attention due to its $(2 \times 2 \times 2)$ charge-density-wave (CDW) transition with periodic lattice distortions at a critical temperature $T_{\mathrm{C}} \sim 202 \mathrm{~K}$ [23]. CDW transition has been extensively studied in several 2D TMDs, recently. For instance, layered $\mathrm{TaS}_{2}$, [24] $\mathrm{TaSe}_{2}$ [25] and $\mathrm{NbSe}_{2}$ [26] have been synthesized and their transition temperatures to the $\mathrm{CDW}$ phase have been reported. $\mathrm{CDW}$ phase in the single layer form of $\mathrm{TiSe}_{2}$ is more robust than that of bulk with an elevated $T_{\mathrm{C}} \sim 232 \mathrm{~K}$ [27]. The transition is 
attributed to band structure effect stemming from energy minimization. Angle-resolved photoemission spectroscopy (ARPES) measurements show that single-layer $\mathrm{TiSe}_{2}$ has a temperature-tunable small band gap at room temperature and the gap increases with decreasing temperature [27]. Fang et al showed that distortions of $\mathrm{Ti}$ and Se atoms in the CDW phase of $\mathrm{TiSe}_{2}$ are related to their atomic mass ratio [28].

To functionalize 2D materials, many strategies have been proposed, such as applying strain, $[29,30]$ fabrication of their nanoribbons, [31, 32] implanting impurities and vacancies $[33,34]$. For example, Nair et al synthesized fluorographene by exposing of graphene to atomic F[35]. It was found that fluorographene is an insulator with an optical gap of $3 \mathrm{eV}$. Produced fluorographene shows similar mechanical properties with graphene. Sahin et al have reported possible chlorinated graphene derivatives [36]. It was found that two-face chlorinated graphene is stable and it is a direct gap semiconductor [36]. Hydrogen is commonly used to functionalize 2D materials. For example, Elias et al have found that compared to pristine graphene, the electronic properties of hydrogenated graphene (graphane) change drastically [37]. Moreover, it was shown that the reaction with hydrogen is reversible, thus distinctive properties of graphene can be restored. In the recent study of Bacaksiz et al, the interaction between $\mathrm{H}$ atom and $\mathrm{PbI}_{2}$ surface was studied [38]. It was reported that $(2 \times 1)$ and $(2 \times 2)$ JahnTeller type distortions occur for the half-hydrogenated and full hydrogenated $\mathrm{PbI}_{2}$, respectively. These reconstructions lead to significant modifications on the electronic and magnetic properties of the material.

Motivated by these studies, in this paper, structural, phononic, thermal and electronic properties of pristine and fully hydrogenated single-layer $\mathrm{TiSe}_{2}$ were studied by using density functional theory (DFT) based calculations. It was found that $\mathrm{TiSe}_{2}$ has a distorted CDW phase in its ground state. The phase transition from distorted CDW phase to $T_{d}$ phase is observed via hydrogenation of $\mathrm{TiSe}_{2}$. In addition, the effect of hydrogenation on the characteristic properties of single-layer $\mathrm{TiSe}_{2}$ was investigated.

\section{Computational methodology}

First principle calculations were performed using the spinpolarized DFT and the projector augmented wave [39, 40] method, as implemented in the Vienna $a b$ initio Simulation Package [41, 42]. The electronic exchange-correlation potential was treated within the generalized gradient approximation (GGA) of Perdew-Burke-Ernzerhof (PBE) [43]. For a better approximation of band gap values underestimated by PBE functional, the Heyd-Scuseria-Ernzerhof (HSE06) hybrid functional was also used [44]. In the HSE06 approach, the fraction of the Hartree-Fock exchange and the screening parameter were set to $\alpha=0.25$ and $0.2 \AA^{-1}$, respectively.

The conjugate gradient algorithm was used to optimize the structure. A plane-wave basis set with kinetic energy cutoff of $400 \mathrm{eV}$ was used for all the calculations. The convergence criterion for energy was taken to be $10^{-5} \mathrm{eV}$ between two consecutive steps. All the structures were fully optimized to minimize each component of the interatomic Hellmann-Feynman forces until a precision of $10^{-4} \mathrm{eV}^{-1}$ was reached. To hinder interactions between the adjacent cells, at least $12 \AA$ vacuum space was used along the $z$-direction. The vdW correction to the GGA functional was included by using the DFT-D2 method of Grimme [45]. Brillouin Zone integration was performed using $12 \times 12 \times 1$ and $18 \times 9 \times 1 \Gamma$-centered k-point meshes for the the density of state calculations of pristine and fully hydrogented $\mathrm{TiSe}_{2}$, respectively. Phonon calculations were performed by making use of the small displacement method as implemented in the PHON code [46].

\section{Pristine $\mathrm{TiSe}_{2}$}

\subsection{Structural properties}

A single-layer $\mathrm{TiSe}_{2}$ consists of stacked Se-Ti-Se atomic layers. $\mathrm{Ti}$ and $\mathrm{Se}$ atoms are strongly bound within the layer. Recent ARPES and scanning tunneling microscopy measurements revealed that single-layer $\mathrm{TiSe}_{2}$ exhibits a $(1 \times 1)$ $1 \mathrm{~T}$ phase at room temperature and a $(2 \times 2) \mathrm{CDW}$ phase at low temperatures [22, 47].

The optimized atomic structures of $1 \mathrm{~T}$ and CDW phases of $\mathrm{TiSe}_{2}$ are shown in figure 1(a). The lattice parameters of the optimized crystal structures of $(1 \times 1) 1 \mathrm{~T}$ and $(2 \times 2)$ CDW phases are 3.50 and $7.00 \AA$, respectively. 1T phase belongs to $\mathrm{P} \overline{3} \mathrm{~m} 1$ space group, with an hexagonal Bravais lattice. The bond distance between $\mathrm{Ti}$ and $\mathrm{Se}$ atoms for $1 \mathrm{~T}$ phase is $2.55 \AA$. On the other hand, CDW phase exhibits periodic lattice distortions and $\mathrm{Ti}-\mathrm{Se}$ bond lengths vary from 2.49 to $2.63 \AA$. There are two types of $\mathrm{Ti}$ (Se) atoms in the unit cell. While one of $\mathrm{Ti}(\mathrm{Se})$ remains fixed, the other is displaced to form the lattice distortions. Fixed and displaced $\mathrm{Ti}(\mathrm{Se})$ atoms in the unit cell are labeled as $\mathrm{Ti}_{f}\left(\mathrm{Se}_{f}\right)$ and $\mathrm{Ti}_{d}$ $\left(\mathrm{Se}_{d}\right)$, respectively (shown in figure 3(a)). Proportional magnitudes and directions of atomic displacements of displaced $\mathrm{Ti}$ and Se are shown by dark blue and orange vectors in figure 1(a). The calculated atomic displacements of $\mathrm{Ti}$ and $\mathrm{Se}$ atoms are $0.09 \AA$ and $0.03 \AA$, respectively. To determine the most favorable structure of single-layer $\mathrm{TiSe}_{2}$, the total energies of 1T phase and CDW phase for the same number of atoms are calculated. It is found that CDW phase is energetically the most favorable structure, which has $4 \mathrm{meV}$ lower energy per formula unit than the $1 \mathrm{~T}$ phase.

\subsection{Phononic properties}

Vibrational properties are critical for the analysis of dynamic stability of a material. The phonon dispersions of $1 \mathrm{~T}$ and CDW phases of single-layer $\mathrm{TiSe}_{2}$ are shown in figures 1 (b) and (c), respectively. The unit cells of $1 \mathrm{~T}$ and CDW phases consist of 3 and 12 atoms, respectively. Therefore, the phonon dispersion of the $1 \mathrm{~T}$ phase yields 3 acoustic and 6 optical modes, whereas that of CDW phase possesses 3 acoustic and 33 optical modes. The force constant matrix is calculated by displacing atoms from their equilibrium positions in $6 \times 6 \times 1$ and $3 \times 3 \times 1$ 

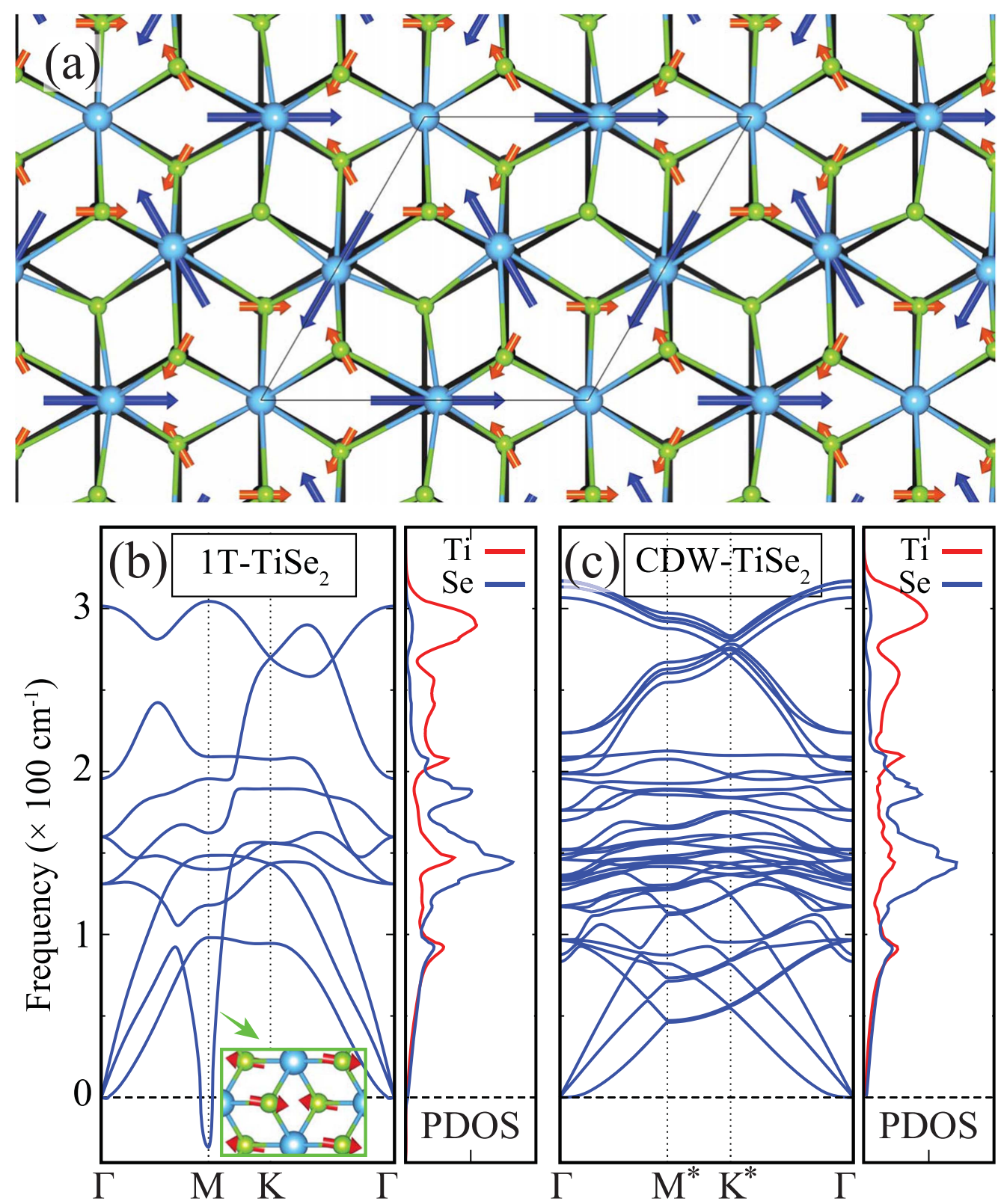

Figure 1. (a) Top view of CDW phase of single-layer TiSe $e_{2}$. Black lattice in the background illustrates the 1T phase of TiSe $e_{2}$. Light blue and green balls illustrate Ti and Se atoms, respectively. Dark blue and orange vectors show proportional magnitudes and directions of the relevant atomic displacements. Phonon band diagrams and partial phonon DOS of (b) 1T phase (Inset: top view of atomic displacements of the corresponding mode) and (c) CDW phase of single-layer $\mathrm{TiSe}_{2}$.

supercells for the $(1 \times 1) 1 \mathrm{~T}$ and $(2 \times 2)$ CDW phases, respectively.

Figure 1(b) shows that $1 \mathrm{~T}$ phase is dynamically unstable with a Kohn-type soft phonon mode at the $M$ high symmetry point [48]. In order to further understand the instability of the $1 \mathrm{~T}$ phase, atomic displacements of the soft phonon mode are shown in the inset of figure 1(b). It is seen that the mode responsible for the instability of the $1 \mathrm{~T}$ phase is due to inplane motion of Se atoms towards to $\mathrm{Ti}-\mathrm{Ti}$ bonding center. Imaginary eigenfrequencies at the $M$ high symmetry point indicate the lack of required restoring force against the motion of Se atoms towards to Ti-Ti bonding center, and they can be cured by a structural phase transition from $1 \mathrm{~T}$ phase to CDW phase. As seen from figure $1(\mathrm{c})$, phonon spectra of $(2 \times 2)$
CDW phase have positive eigenfrequencies in the whole Brillouin Zone that indicate the dynamical stability of the CDW phase. As seen in figure 1(c), distortions of the CDW phase lead to almost flat phonon bands near the $200 \mathrm{~cm}^{-1}$. It is known that materials with hexagonal (or trigonal) crystal symmetry exhibit linear crossing phonon branches at the $K$ high symmetry point. As clear from figure 1(a), despite the distorted structure, trigonal symmetry of the CDW phase is protected. Therefore, phonon dispersion of the CDW phase exhibits the linear crossing at the $K^{*}$ high symmetry point (shown in figure 1(c)).

In addition, partial phonon density of states of $1 \mathrm{~T}$ and CDW phases are also shown in figure 1. For both phases, the low-frequency modes, below $100 \mathrm{~cm}^{-1}$, originate from 


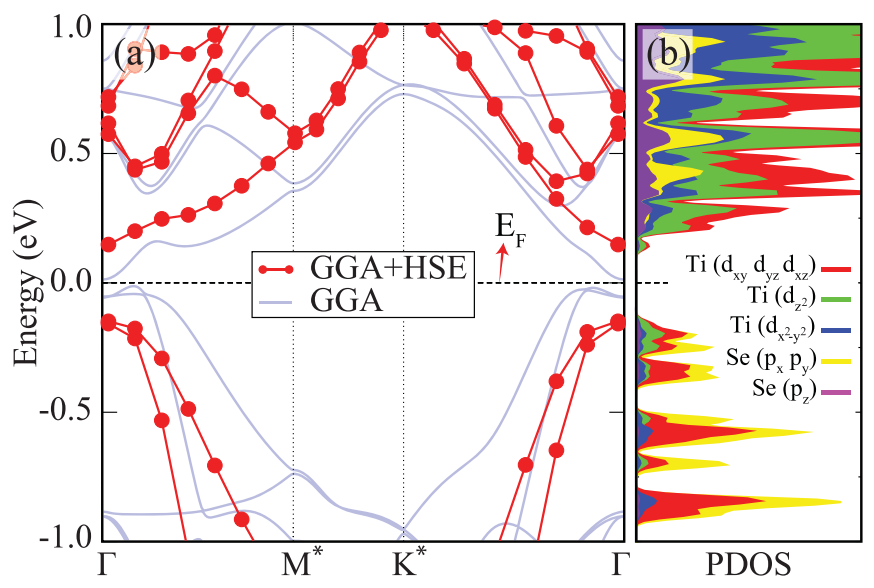

Figure 2. (a) The energy-band structure of CDW phase of $\mathrm{TiSe}_{2}$. Light blue and red-dotted bands are for GGA and GGA+HSE, respectively. (b) GGA+HSE calculated partial density of states of $\mathrm{CDW}$ phase of $\mathrm{TiSe}_{2}$. The valence band maximum is set to zero.

vibrations of the $\mathrm{Ti}$ and $\mathrm{Se}$ atoms. The medium-frequency modes, $100-200 \mathrm{~cm}^{-1}$, are dominated by vibrations of the Se atom, whereas the high-frequency modes, above $200 \mathrm{~cm}^{-1}$, are mainly composed of vibrations of the $\mathrm{Ti}$ atom, since atomic mass of Se is higher than Ti.

\subsection{Electronic properties}

The electronic band structure and partial density of states (PDOS) of CDW phase of single-layer $\mathrm{TiSe}_{2}$ are shown in figure 2. The band gap of $\mathrm{TiSe}_{2}$ is calculated to be $25 \mathrm{meV}$ by using the PBE approximation. Since the bare PBE functional usually underestimates the band gap of semiconducting materials, the HSE06 functional is used to get a more precise band gap. Therefore, $\mathrm{TiSe}_{2}$ is found to be a direct gap semiconductor with a band gap of $319 \mathrm{meV}$ with HSE06 functional. The valence band maximum (VBM) and conduction band minimum $(\mathrm{CBM})$ reside at the $\Gamma$ point, as shown in figure 2. The calculated electronic band structure within HSE06 agrees well with previous experimental and theoretical results [22, 27].

Previous studies showed that $p-d$ orbital hybridizations play an important role in determining the electronic and structural characteristics of $4 d$-TMDs [49]. Therefore, these orbital interactions deserve considerable attention to determine exact electronic structure of single-layer $\mathrm{TiSe}_{2}$. Calculated PDOS reveals that the CBM is made up of the $t_{2 g}\left(d_{x y}\right.$, $\left.d_{y z}, d_{z x}\right)$ and $d_{z^{2}}$ orbitals of Ti atoms. The states in the vicinity of VBM are mainly composed of hybridization of $t_{2 g}$ and $d_{z^{2}}$ orbitals of Ti atom and $p_{x}$ and $p_{y}$ orbitals of Se atom. Near the Fermi level, overlap of PDOS peak positions and shapes of $p$ orbitals of $\mathrm{Se}$ atom and $d$ orbitals of $\mathrm{Ti}$ atom reveal strong coupling of these orbitals.

In $4 d$-TMDs (such as $(1 \times 1) 1 \mathrm{~T}$ phase of $\left.\mathrm{TiSe}_{2}\right) \mathrm{Ti}$ atoms can interact with each other through intermetal $t_{2 g}$ orbitals. Due to weak coupling of these orbitals, $t_{2 g}$ states are mostly located near the Fermi level, with no energy gap.
Differing from the $(1 \times 1) 1 \mathrm{~T}$ phase, periodic lattice distortions of the $(2 \times 2) \mathrm{CDW}$ phase lead to increase in the intermetal $t_{2 g}$ orbital interactions. Furthermore, excess electrons of Se atoms lead to attractive interaction in the $t_{2 g}-p$ orbitals. Therefore, Se atoms are exposed to a force towards to Ti-Ti bonding center and occupied $p$ states of the Se atom shift to lower energies. As a result, CDW phase transition has a significant effect on the electronic structure and leads to opening of a band gap in $\mathrm{TiSe}_{2}$.

\section{Interaction with a single $\mathrm{H}$ atom}

\subsection{Structural and electronic properties}

Understanding of the adsorption properties of a single $\mathrm{H}$ atom on $\mathrm{TiSe}_{2}$ is crucial to the investigation of its hydrogenated derivatives. We simulate the adsorption of a $\mathrm{H}$ atom on a CDW phase of single-layer $\mathrm{TiSe}_{2}$ by considering six inequivalent adsorption sites, as illustrated in figure 3 (a). A $2 \times 2$ supercell of $\mathrm{TiSe}_{2}$ containing $16 \mathrm{Ti}$ and $32 \mathrm{Se}$ is used to hinder $\mathrm{H}-\mathrm{H}$ interaction. The nearest distance between $\mathrm{H}$ adatoms is larger than $14 \AA$. The atomic positions were fully relaxed starting from the six different adatom initial configurations: $\mathrm{Ti}_{f}, \mathrm{Ti}_{d}, \mathrm{Se}_{f}^{t}, \mathrm{Se}_{f}^{b}, \mathrm{Se}_{d}^{t}$, and $\mathrm{Se}_{d}^{b}$ as shown in figure 3 . The binding energy of a $\mathrm{H}$ atom is calculated as

$$
E_{b}=E_{\mathrm{TiSe}_{2}}+E_{\mathrm{H}}-E_{\mathrm{TiSe}_{2}+\mathrm{H}}
$$

where $E_{\mathrm{TiSe}_{2}}$ denotes the energy of the $(2 \times 2)$ supercell of $\mathrm{CDW}$ phase of single-layer $\mathrm{TiSe}_{2}, E_{\mathrm{H}}$ is the energy of isolated $\mathrm{H}$ atom, and $E_{\mathrm{TiSe}_{2}+\mathrm{H}}$ denotes the total energy when the $\mathrm{H}$ is adsorbed on $\mathrm{TiSe}_{2}$.

Calculated lattice constants, binding energies, and the equilibrium distance between the adatom and the nearest Se atom are given in table 1. It is found that the binding energy of $\mathrm{H}$ atom is $1.69 \mathrm{eV}$ for both configuration $\mathrm{H}$ on top of $\mathrm{Se}_{f}^{t}$ or $\mathrm{Se}_{d}^{t}$. These sites are energetically the most favorable ones. When a single $\mathrm{H}$ adsorption is introduced, the $2 \times 2$ supercell of $\mathrm{TiSe}_{2}$ enlarges from 14.00 to $14.08 \AA$. As shown in figure $3(\mathrm{~b})$, the adsorbed $\mathrm{H}$ atom modifies the local atomic structure around it. The nearest $\mathrm{Se}-\mathrm{Ti}$ bonds to the adsorbed atom are increased from $2.49,2.56$, and $2.63 \AA$ to $2.63,2.63$, and $2.64 \AA$, respectively. The distance between $\mathrm{H}$ and $\mathrm{Se}$ atoms is $1.48 \AA$.

Energy-band dispersion and PDOS of a $\mathrm{H}$ atom-adsorbed single-layer $\mathrm{TiSe}_{2}$ are shown in figure 3(c). When single $\mathrm{H}$ atom is adsorbed on $\mathrm{TiSe}_{2}$, the structure becomes metallic. Although $\mathrm{H}$ atom has a major effect on the electronic structure of $\mathrm{TiSe}_{2}$, the states originating from $\mathrm{H}$ atom reside at deep energy levels, as shown in figure 3(c). The states around the Fermi level are mainly composed of $d$ orbitals of $\mathrm{Ti}$ atoms. The presence of a $\mathrm{H}$ adatom does not change the nonmagnetic character of $\mathrm{TiSe}_{2}$. 

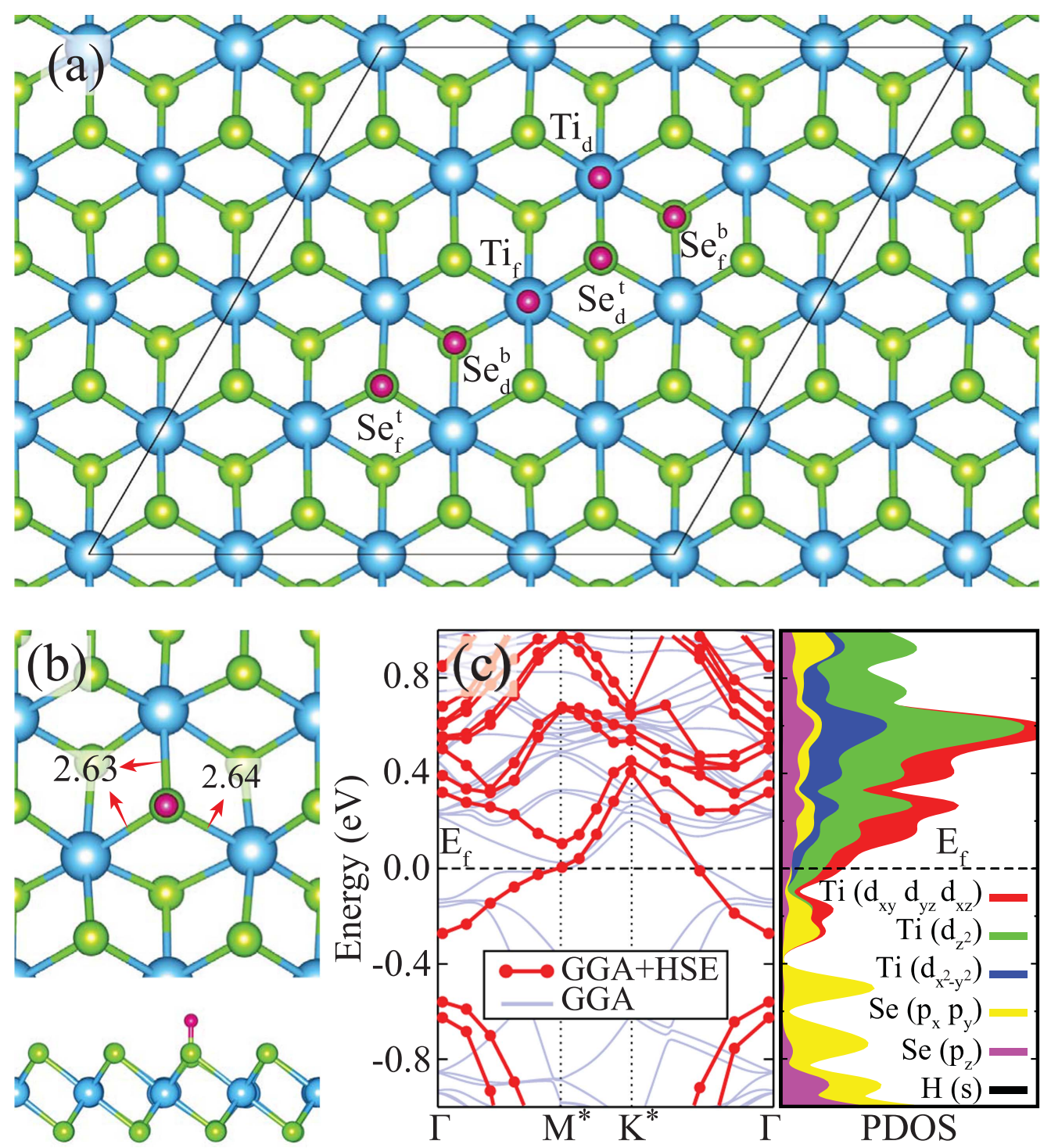

Figure 3. (a) Top view of six inequivalent adsorption configurations for a $\mathrm{H}$ atom on CDW phase of TiSe 2 . (b) Top and side views of fully relaxed geometric structure of single $\mathrm{H}$ atom adsorbed $\mathrm{CDW}$ phase of $\mathrm{TiSe}_{2}$. (c) The electronic band structure and PDOS of single $\mathrm{H}$ atom adsorbed CDW phase of $\mathrm{TiSe}_{2}$. Light blue and red-dotted bands are for GGA and GGA+HSE, respectively.

Table 1. The lattice constants, $a$ and $b$; the distance from adsorbed $\mathrm{H}$ atom to the $\mathrm{TiSe}_{2}$ surface, $d_{\mathrm{Se}-\mathrm{H}}$; binding energy, $E_{b}$; HSE calculated electronic band gap, $E_{g}$; of CDW phase, single-H adsorbed and fully hydrogenated $\mathrm{TiSe}_{2}\left(\mathrm{fH}-\mathrm{TiSe}_{2}\right)$.

\begin{tabular}{lccccc}
\hline & $\begin{array}{c}a \\
(\AA)\end{array}$ & $\begin{array}{c}b \\
(\AA)\end{array}$ & $\begin{array}{c}d_{\mathrm{Se}-\mathrm{H}} \\
(\AA)\end{array}$ & $\begin{array}{c}E_{b} \\
(\mathrm{eV})\end{array}$ & $\begin{array}{c}E_{g} \\
(\mathrm{meV})\end{array}$ \\
\hline CDW phase & 7.00 & 7.00 & - & - & 319 \\
Single-H on $\mathrm{TiSe}_{2}$ & 14.08 & 14.08 & 1.48 & 1.69 & - \\
fH-TiSe $_{2}$ & 3.61 & 6.50 & $1.53 / 1.49$ & 2.55 & 119 \\
\hline
\end{tabular}

\section{Full hydrogenation}

\subsection{Structural and phononic properties}

Following the analysis of the adsorption of a $\mathrm{H}$ atom on CDW phase of $\mathrm{TiSe}_{2}$, modifications of structural, electronic, phononic and thermal properties of $\mathrm{TiSe}_{2}$ upon full hydrogenation are investigated. To construct a fully covered structure the most favorable position of an isolated $\mathrm{H}$ atom is used. Therefore, fully hydrogenated $\mathrm{TiSe}_{2}$, with each $\mathrm{H}$ atom resides on top of a $\mathrm{Se}$ atom is investigated.

It is found that through full hydrogenation, $\mathrm{TiSe}_{2}$ experiences a structural phase transition from CDW phase to $T_{d}$ phase. Figures 4(a) and (b) show top and side views of the optimized atomic structure of fully hydrogenated single-layer $\mathrm{TiSe}_{2}\left(\mathrm{fH}-\mathrm{TiSe}_{2}\right) . T_{d}$ phase has a rectangular unit cell with calculated lattice constants of $a=3.61 \AA$ and $b=6.50 \AA$. The unit cell of fH-TiSe 2 is composed of two Ti, four Se, and four $\mathrm{H}$ atoms. The hydrogenation of each Se atom leads to a reduced interaction between $\mathrm{Ti}$ and $\mathrm{Se}$ atoms. Therefore, transition metal atoms get closer and form separated zig-zag chains. Ti-Ti bond distance in the zig-zag chain is calculated to be $3.12 \AA$. The bond distance between $\mathrm{Ti}$ and $\mathrm{Se}$ atom is in the range of $2.48-2.76 \AA$. Due to reduced symmetry of the $T_{d}$ phase, there are two different Se atoms: outer-Se and 

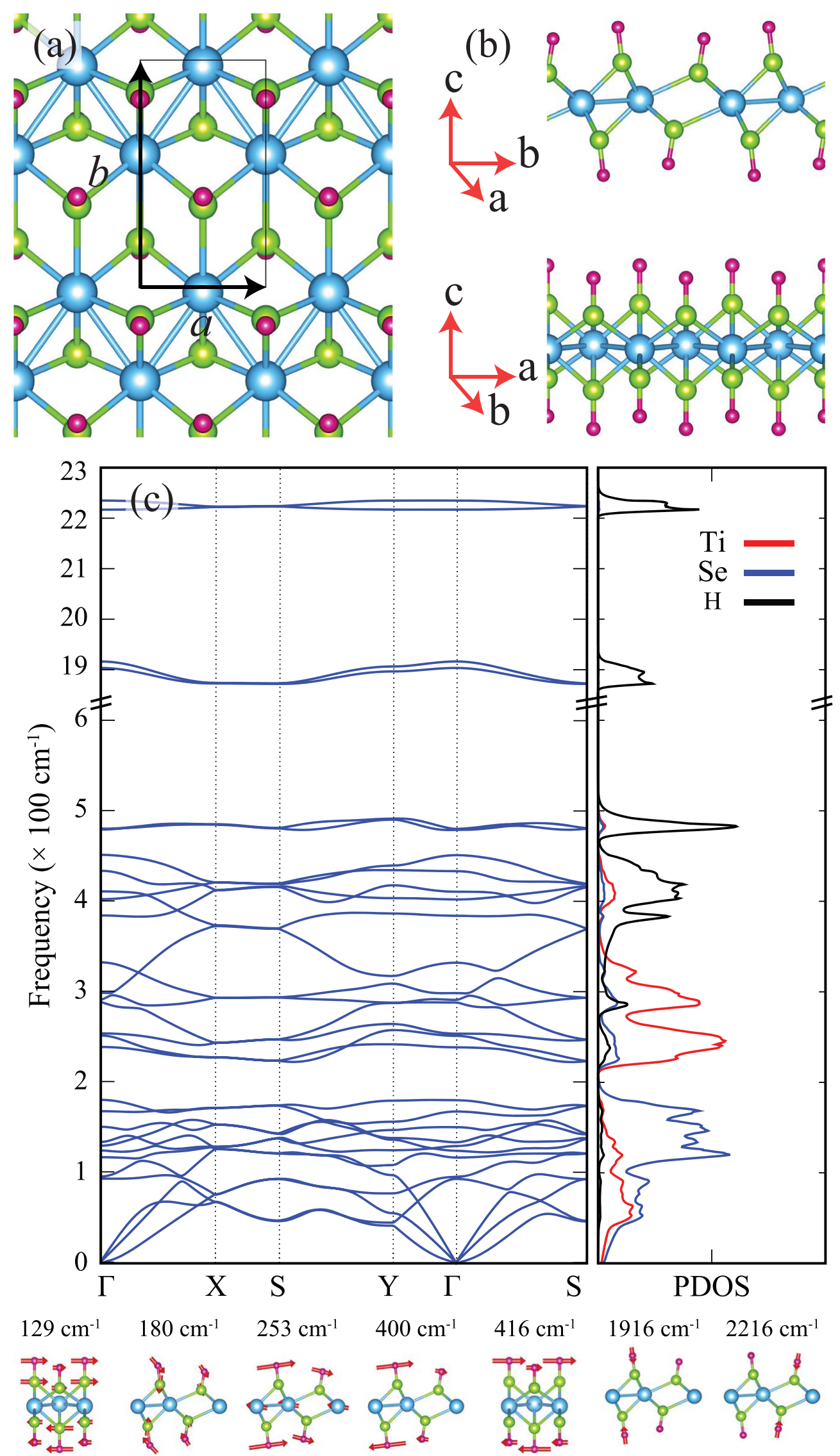

Figure 4. (a) Top and (b) side view geometries and (c) phonon band diagram and partial phonon DOS of fH-TiSe 2 . The branches of the possible Raman-active modes are shown on the lower panel. 
inner-Se. $\mathrm{H}$ atoms reside on top of these Se atoms. The bond length between outer-Se (inner-Se) and $\mathrm{H}$ atom is 1.53 (1.49) $\AA$. The calculated average binding energy of a $\mathrm{H}$ atom is $2.55 \mathrm{eV}$, which is much higher than the binding energy of a $\mathrm{H}$ adsorption case.

Phonon dispersion and partial phonon DOS of the $\mathrm{fH}^{-\mathrm{TiSe}_{2}}$ are shown in figure $4(\mathrm{c}) .4 \times 2 \times 1$ supercell is used for the phonon calculations of $\mathrm{fH}-\mathrm{TiSe}_{2}$. It is found that fully hydrogenated $\mathrm{TiSe}_{2}$ has real vibrational eigenfrequencies in the whole Brillouin zone and hence $\mathrm{fH}-\mathrm{TiSe}_{2}$ is dynamically stable. The unit cell of $\mathrm{fH}-\mathrm{TiSe}_{2}$ consists of 10 atoms, consequently it possesses 30 phonon bands, 3 acoustic and 27 optical. As mentioned above, hydrogenation of $\mathrm{TiSe}_{2}$ induces a significant structural transition from CDW phase to $T_{d}$ phase. Comparison between the phonon calculations of pristine and fully hydrogenated $\mathrm{TiSe}_{2}$ shows significant differences. Unlike the pristine case, the high-frequency optical modes of $\mathrm{fH}-\mathrm{TiSe}_{2}$ are separated from the low-frequency modes by a gap of $42 \mathrm{~cm}^{-1}$. Full hydrogenation causes to presence of optical phonon branches at quite high frequencies. It is seen from the right panel of figure 4(c), above $350 \mathrm{~cm}^{-1}$ the phonon partial DOS almost entirely consists of vibrations of $\mathrm{H}$ atoms.

In figure 4(c) the atomic displacements of possible Raman active modes are also presented. The low frequency characteristic Raman-active mode at $129 \mathrm{~cm}^{-1}$ corresponds to an in-plane $\left(E_{g}\right.$ like) a counter-phase motion of top and bottom Se and $\mathrm{H}$ layers (parallel to the $\vec{a}$ ). The Raman-active mode with frequency of $180 \mathrm{~cm}^{-1}$ corresponds to a mixed in-plane and out-of-plane $\left(A_{g}\right.$ like) counter-phase motion of top and bottom Se and $\mathrm{H}$ layers (parallel to the $\vec{b}$ ). However, the character of the phonon mode at $253 \mathrm{~cm}^{-1}$ is mostly in-plane and while Se atoms stay fixed, $\mathrm{Ti}$ and $\mathrm{H}$ atoms move in opposite directions to each other (parallel to the $\vec{b}$ ). The phonon modes at 400 and $416 \mathrm{~cm}^{-1}$ correspond to in-plane counter-phase motions of top and bottom $\mathrm{H}$ layers (parallel to the $\vec{b}$ and $\vec{a}$, respectively). As can be seen from the figure 4(c), the high-frequency modes at 1916 and $2216 \mathrm{~cm}^{-1}$ are Se-H stretching modes.

\subsection{Heat capacity}

The thermal properties of ultra-thin materials can be investigated through the calculation of heat capacity $\left(C_{v}\right)$. It is known that contribution of free conduction electrons to the heat capacity of ultra-thin materials is negligible. Hence, the heat capacity is constituted almost entirely from lattice vibrations of the material.

In this part we discuss the effect of hydrogenation on the heat capacity of $\mathrm{TiSe}_{2}$. The heat capacities of 1T, CDW phases and fully hydrogenated $\mathrm{TiSe}_{2}$ as a function of temperature are shown in figure 5. Previously, it was shown that hydrogenation of ultra-thin materials leads to an increase in the heat capacity of the materials [50]. However, as seen from figure 5, the heat capacity of fully hydrogenated $\mathrm{TiSe}_{2}$ is always lower than the heat capacity of $1 \mathrm{~T}$ and CDW phases of $\mathrm{TiSe}_{2}$ for all temperatures. Decrease in the heat capacity is attributed to the phonon band gap observed at relatively low frequencies (between 180 and $222 \mathrm{~cm}^{-1}$ ). It is known that the

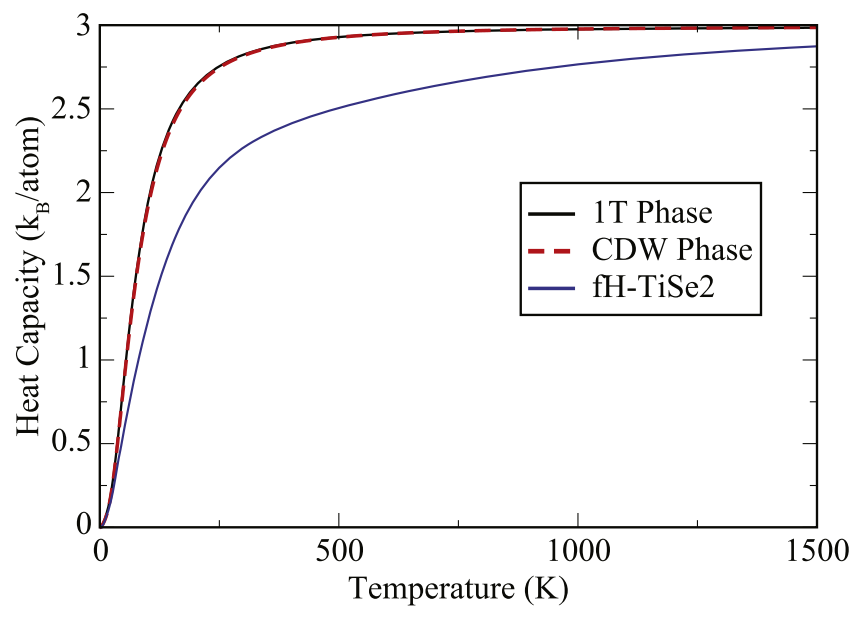

Figure 5. Constant-volume heat capacity of single-layer crystal structures of $1 \mathrm{~T}$ and CDW phases of pristine $\mathrm{TiSe}_{2}$ and $\mathrm{fH}-\mathrm{TiSe}_{2}$.

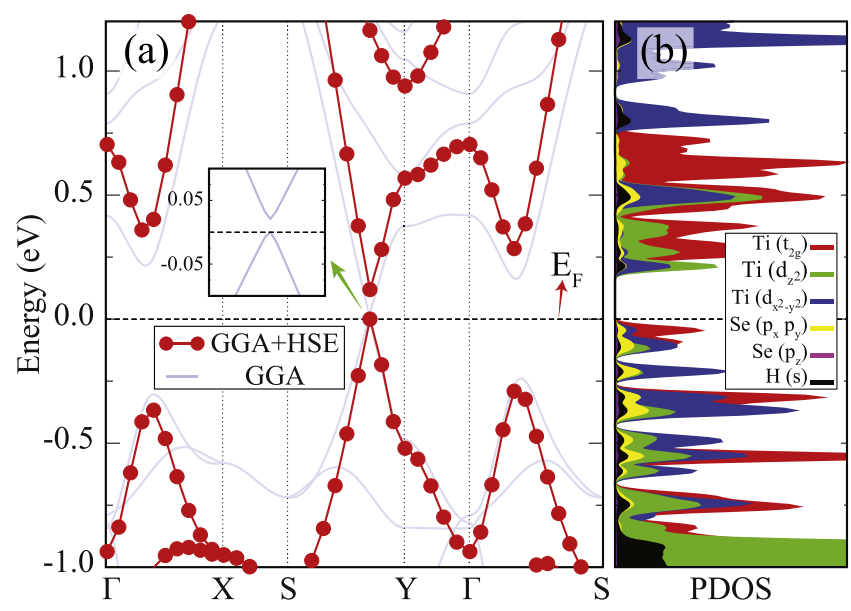

Figure 6. (a) The electronic band structure of fH-TiSe 2 . Light blue and red-dotted bands are for GGA and GGA + HSE calculated band dispersions, respectively. (Inset: GGA calculated band dispersion near the fermi level.) (b) GGA+HSE calculated PDOS of fH-TiSe 2 . The valence band maximum is set to zero.

heat capacity is closely related with the phonon DOS of the material. Lack of phonon DOS at the phonon band gap lead to decrease in the heat capacity of the material. At room temperature, $C_{v}$ of $1 \mathrm{~T}$ and CDW phases are equal to $2.82 k_{B}$ /atom, whereas $C_{v}$ of fH-TiSe2 is $2.27 k_{B}$ /atom. At $1500 \mathrm{~K}$, the heat capacity for $1 \mathrm{~T}$ and CDW phases of $\mathrm{TiSe}_{2}$ approach the value of Dulong-Petit law $C_{v}=3 \mathrm{NR}$, where $R$ is the universal gas constant and $N$ is the number of atoms per unit cell. However, the heat capacity for $\mathrm{fH}-\mathrm{TiSe}_{2}$ approaches to this value at higher temperatures. Calculated Debye temperature values of 1T, CDW phases and $\mathrm{fH}-\mathrm{TiSe}_{2}$ are 335, 341, and $1378 \mathrm{~K}$, respectively.

\subsection{Electronic properties}

The electronic band dispersions and PDOS obtained by using HSE06 functional of $\mathrm{fH}-\mathrm{TiSe}_{2}$ are shown in figures 6(a) and (b), respectively. It is found that hydrogenation of single-layer $\mathrm{TiSe}_{2}$ leads to reduction in the energy band gap. Band gap of fully hydrogenated $\mathrm{TiSe}_{2}$ is calculated to be $119 \mathrm{meV}$ when 
HSE06 functional is used (GGA value: $21 \mathrm{meV}$ ). Although $\mathrm{TiSe}_{2}$ conserves its direct gap semiconducting behavior with hydrogenation, both VBM and CBM transfer to different wavevector point within $Y$ and $S$ from $\Gamma$ point, where VBM and CBM of pristine $\mathrm{TiSe}_{2}$ reside.

Calculated PDOS shows that the states in the VBM are mainly made up of the $t_{2 g}$ orbitals of Ti atom, whereas the states in the CBM have $d_{z^{2}}$ and $d_{x^{2}-y^{2}}$ orbitals of Ti atom. It is clearly seen from figure 6(b), near the Fermi level the contribution comes from $t_{2 g}$ orbital of Ti atom is increased by hydrogenation, while $p$ orbital contribution of Se atom is decreased. Compared to the pristine case, decreased overlap of PDOS peak positions and shapes of $t_{2 g}$ orbitals of Ti atom and $p$ orbitals of $\mathrm{Se}$ atom exhibit weak coupling of these orbitals through hydrogenation. This weak coupling can be attributed to the weakening of the bonds between the $\mathrm{Ti}$ and $\mathrm{Se}$ atoms due to the binding of $\mathrm{H}$ atoms to each $\mathrm{Se}$ atom. Therefore, $\mathrm{Ti}$ atoms are released, they move toward to each other. This leads to a structural phase transition from the CDW phase to the $T_{d}$ phase.

\section{Conclusions}

In summary, the structural, phononic, thermal, and electronic properties of pristine and hydrogenated single-layer $\mathrm{TiSe}_{2}$ were investigated within first-principles DFT calculations. It was found that the periodic lattice distortions in the CDW phase provide the stability of the layer. Our calculations showed that experimentally fabricated low temperature phase (CDW phase) of $\mathrm{TiSe}_{2}$ has an HSE06-calculated direct band gap of $319 \mathrm{meV}$.

In addition, adsorption of one $\mathrm{H}$ atom on the $\mathrm{CDW}$ phase of $\mathrm{TiSe}_{2}$ was investigated. Preferred adsorption site, binding energy and electronic properties of single-H adsorbed CDW phase of $\mathrm{TiSe}_{2}$ were determined. Moreover, our calculations revealed a structural transition resulting from the rearrangement of the Ti atoms after full hydrogenation of single-layer $\mathrm{TiSe}_{2}$. The fully hydrogenated $\mathrm{TiSe}_{2}$ prefers $\mathrm{T}_{d}$ phase which exhibits Ti-Ti dimerization along one of the lattice parameters. Structural stability of the fully hydrogenated $\mathrm{TiSe}_{2}$ was confirmed by calculated phonon spectra of the layer. Direct electronic band gap of the material decreased to 119 $\mathrm{meV}$ upon full hydrogenation. Furthermore, it was found that full hydrogenation of $\mathrm{TiSe}_{2}$ leads to a dramatic decrease in the heat capacity. Single-layer $\mathrm{TiSe}_{2}$ with robust semiconducting character, sensitive thermal properties, and hydrogenationinduced structural transition is highly desired material for nanoscale device applications.

\section{Acknowledgments}

Computational resources were provided by TUBITAK ULAKBIM, High Performance and Grid Computing Center (TR-Grid e-Infrastructure). FI, RTS and HS acknowledge the support from TUBITAK project number 114F397. HS acknowledges financial support from the TUBITAK under the project number 116C073. HS acknowledges support from Bilim Akademisi-The Science Academy, Turkey under the BAGEP program.

\section{ORCID iDs}

F Iyikanat (iD https://orcid.org/0000-0003-1786-3235

A Kandemir (ii) https://orcid.org/0000-0001-9813-6421

R T Senger (iD https://orcid.org/0000-0003-0800-1924

H Sahin (1) https://orcid.org/0000-0002-6189-6707

\section{References}

[1] Novoselov K S, Geim A K, Morozov S V, Jiang D, Zhang Y, Dubonos S V, Grigorieva I V and Firsov A A 2004 Science 306666

[2] Geim A K and Novoselov K S 2007 Nat. Mater. 6183

[3] Pacile D, Meyer J C, Girit C O and Zettl A 2008 Appl. Phys. Lett. 92133107

[4] Han W Q, Wu L, Zhu Y, Watanabe K and Taniguchi T 2008 Appl. Phys. Lett. 93223103

[5] Kim K K et al 2012 Nano Lett. 12161

[6] Cahangirov S, Topsakal M, Akturk E, Sahin H and Ciraci S 2009 Phys. Rev. Lett. 102236804

[7] Vogt P, Padova P D, Quaresima C, Avila J, Frantzeskakis E, Asensio M C, Resta A, Ealet B and Lay G L 2012 Phys. Rev. Lett. 108155501

[8] Gordon R A, Yang D, Crozier E D, Jiang D T and Frindt R F 2002 Phys. Rev. B 65125407

[9] Mak K F, Lee C, Hone J, Shan J and Heinz T F 2010 Phys. Rev. Lett. 105136805

[10] Coleman J N et al 2011 Science 331568

[11] Sahin H, Tongay S, Horzum S, Fan W, Zhou J, Li J, Wu J and Peeters F M 2013 Phys. Rev. B 87165409

[12] Ross J S et al 2014 Nat. Nanotechnol. 9268

[13] Chen B, Sahin H, Suslu A, Ding L, Bertoni M I, Peeters F M and Tongay S 2015 ACS Nano 95326

[14] Ferrer I J, Ares J R, Clamagirand J M, Barawi M and Sanchez C 2013 Thin Solid Film 535398

[15] Pant A et al 2016 Nanoscale 816259

[16] Dai J, Li M and Zeng X C 2016 Wiley Interdiscip. Rev.: Comput. Mol. Sci. 6211

[17] Kim S et al 2012 Nat. Commun. 31011

[18] Podzorov V and Gershenson M E 2004 Appl. Phys. Lett. 843301

[19] Zhang W, Huang Z, Zhang W and Li Y 2014 Nano Res. 71731

[20] Wang Q H, Zadeh K K, Kis A, Coleman J N and Strano M S 2012 Nat. Nanotechnol. 7699

[21] Ruppert C, Aslan O B and Heinz T F 2014 Nano Lett. 146231

[22] Sugawara K, Nakata Y, Shimizu R, Han P, Hitosugi T, Sato T and Takahashi T 2016 ACS Nano 101341

[23] Di Salvo F J, Moncton D E and Waszczak J V 1976 Phys. Rev. B 144321

[24] Ritschel T, Trinckauf J, Koepernik K, Buchner B, Zimmermann M, Berger H, Joe Y I, Abbomonte P and Geck J 2015 Nat. Phys. 11328

[25] Samnakay R, Wickramaratne D, Pope T R, Lake R K, Salguero T T and Balandin A A 2015 Nano Lett. 152965

[26] Xi X, Zhao L, Wang Z, Berger H, Forro L, Shan J and Mak K F 2015 Nat. Nanotechnol. 10765

[27] Chen P, Chan Y-H, Fang X-Y, Zhang Y, Chou M Y, Mo S-K, Hussain Z, Fedorov A-V and Chiang T-C 2015 Nat. Commun. 68943 
[28] Fang X, Hong H, Chen P and Chiang T-C 2017 Phys. Rev. B 95201409

[29] Johari P and Shenoy V B 2012 ACS Nano 65449

[30] Song S, Keum D H, Cho S, Perello D, Kim Y and Lee Y H 2016 Nano Lett. 16188

[31] Yagmurcukardes M, Peeters F M, Senger R T and Sahin H 2016 Appl. Phys. Rev. 3041302

[32] Chen Y X, Cui P, Ren X B A, Zhang C D, Jin C H, Zhang Z y and Shih C K 2017 Nat. Commun. 815135

[33] Komsa H P, Kotakoski J, Kurasch S, Lehtinen O, Kaiser U and Krasheninnikov A V 2012 Phys. Rev. Lett. 109035503

[34] Iyikanat F, Sahin H, Senger R T and Peeters F M 2014 APL Mater. 2092801

[35] Nair R R et al 2010 Small 62877

[36] Sahin H and Ciraci S 2012 J. Phys. Chem. C 11624075

[37] Elias D C et al 2009 Science 323610
[38] Bacaksiz C and Sahin H 2016 RSC Adv. 689708

[39] Kresse G and Joubert D 1999 Phys. Rev. B 591758

[40] Blöchl P E 1994 Phys. Rev. B 5017953

[41] Kresse G and Hafner J 1993 Phys. Rev. B 47558

[42] Kresse G and Furthmuller J 1996 Phys. Rev. B 5411169

[43] Perdew J P, Burke K and Ernzerhof M 1996 Phys. Rev. Lett. 773865

[44] Heyd J, Scuseria G and Ernzerhof M 2003 J. Chem. Phys. 1188207

[45] Grimme S 2006 J. Comput. Chem. 271787

[46] Alfe D 2009 Comput. Phys. Commun. 1802622

[47] Peng J-P, Guan J-Q, Zhang H-M, Song C-L, Wang L, He K, Xue Q-K and Ma X-C 2015 Phys. Rev. B 91121113

[48] Kohn W 1959 Phys. Rev. Lett. 29

[49] Yu L, Yan Q and Ruzsinszky A 2017 Nat. Commun. 815224

[50] Neek-Amal M and Peeters F M 2015 Phys. Rev. B 83235437 